\title{
Examples of Aggregation Operators on Membership Degrees of Type-2 Fuzzy Sets
}

\author{
Susana Cubillo $^{1}$ Pablo Hernández ${ }^{2}$ Carmen Torres-Blanc $^{1}$ \\ ${ }^{1}$ Departamento de Matemática Aplicada, Univ. Politécnica de Madrid, \\ 28660 Boadilla del Monte, Madrid, España, \{scubillo,ctorres\} @ fi.upm.es \\ ${ }^{2}$ Departamento de Matemática y Física, Univ. Nacional Experimental del Táchira, \\ San Cristóbal, Táchira, Venezuela, phernandezv@unet.edu.ve
}

\begin{abstract}
Z. Takáč in [16] introduced the aggregation operators on any subalgebra of $\mathbf{M}$ (set of all fuzzy membership degrees of the type- 2 fuzzy sets, that is, the functions from $[0,1]$ to $[0,1])$. Furthermore, he applied the Zadeh's extension principle (see [24]) to obtain in [16, 17] a set of aggregation operators on $\mathbf{L}^{*}$ (the strongly normal and convex functions of $\mathbf{M}$ ). In this paper, we introduce the aggregation operators on any partially ordered and bounded set (poset). This will allow us to suitably provide aggregation operators on $\mathbf{M}$. In this sense, firstly we define a set of operators on $\mathbf{M}$, more general than those given by Z. Takáč, studying some of their properties. Secondly, we focus on some operators obtained through a very different way, proving that they are aggregation operators on $\mathbf{L}$ (set of normal and convex functions of $\mathbf{M})$, and on $\mathbf{M}$.
\end{abstract}

Keywords: Type- 2 fuzzy sets, functions from $[0,1]$ to $[0,1]$, normal and convex functions, aggregation operators

\section{Introduction}

Type-2 fuzzy sets (T2FSs) were introduced by L.A. Zadeh in 1975 [24] as an extension of type-1 fuzzy sets (FSs). Whereas for FSs the membership degree of an element of a set is determined by a value in the interval $[0,1]$, the membership degree of an element for T2FSs is a fuzzy set in $[0,1]$, that is, a T2FS is determined by a membership function $\mu: X \rightarrow \mathbf{M}$, where $\mathbf{M}=[0,1]^{[0,1]}$ is the set of functions from $[0,1]$ to $[0,1]$ (see [11], [14], [15], [19]). In this paper we will get results for T2FSs with membership degrees in $\mathbf{M}=[0,1]^{[0,1]}$ (set of functions from $[0,1]$ to $[0,1]$ ) and also in the subset $\mathbf{L}$ of normal and convex functions of $\mathbf{M}$. Because the membership degree of T2FSs is fuzzy, they are better able to model uncertainty than FSs [12].

Aggregation of information is critical in any inference system, and so the study of aggregation operators is essential, for the fuzzy sets and for any of their extensions, such as type-2 fuzzy sets. The theory of aggregation of real numbers is well established (see e.g. $[13,3,21]$ ) and is applied in fuzzy logic systems based on FSs. Aggregation operators for real numbers were extended to the aggregation operators for intervals (see e.g. [2]). Then,
Z. Takáč $[16,17]$ introduced the definition of aggregation operator on $\mathbf{M}$ and applied the Zadeh's extension principle in order to extend the type-1 aggregation operators to T2FSs. Nevertheless, S. Zhou et al. [25] had previously given an approximation with the extension of the ordinary aggregation operators so-called OWA (Ordered Weighted Averaging, see [22]).

The purpose of this paper is to introduce a family of operators on $\mathbf{M}$, more general than that given in $[16,17]$ and study some of their properties in order to determine under which conditions these operators are aggregation operators on L. Furthermore, we analyze other set of operators on $\mathbf{M}$, different to those obtained in [16] and [17].

The article is organized as follows. Section 2 is devoted to remember the basic concepts to understand the rest of the paper. In Section 3 we study some aggregation operators obtained through the Zadeh's extension principle, while Section 4 focus on a new family of operators. Finally, some conclusions are presented in Section 5.

\section{Preliminaries}

In this Section, we will recall some concepts and results, in order to understand without difficulty the rest of the paper. Throughout the paper, $X$ will denote a non-empty set which will represent the universe of discourse. Additionally, $\leq$ will denote the usual order relation in the lattice of real numbers.

\subsection{Fuzzy sets, some extensions and operations}

Definition 1. ([23]) A type-1 fuzzy set (FS), A, is characterized by a membership function $\mu_{A}$,

$$
\mu_{A}: X \rightarrow[0,1]
$$

where $\mu_{A}(x)$ is the membership degree of an element $x \in X$ in the $\operatorname{set} A$.

Definition 2. ([1], [18]) An interval-valued fuzzy set (IVFS), $A$, is characterized by a membership function $\sigma_{A}$,

$$
\sigma_{A}: X \rightarrow I([0,1])=\{[a, b]: 0 \leq a \leq b \leq 1\} .
$$

Accordingly, the membership degree of an element $x \in$ $X$ in the set $A$ is an interval in $[0,1]$. 
A partial order $\leq_{I}$ can be defined on $I([0,1])$ such that $\left[a_{1}, a_{2}\right] \leq_{I}\left[b_{1}, b_{2}\right]$ if and only if $a_{1} \leq b_{1}$ and $a_{2} \leq b_{2}$. Thus $\left(I([0,1]), \leq_{I}\right)$ is a bounded and complete lattice in which the least and greatest elements are, respectively, $[0,0]$ and $[1,1]$.

Definition 3. ([14], [15]) A type-2 fuzzy set (T2FS), A, is characterized by a membership function:

$$
\mu_{A}: X \rightarrow \mathbf{M}=[0,1]^{[0,1]}=\operatorname{Map}([0,1],[0,1]),
$$

that is, $\mu_{A}(x)$ is a fuzzy set on the interval $[0,1]$ and also the membership degree of an element $x \in X$ in the set $A$. Therefore,

$$
\mu_{A}(x)=f_{x}
$$

where

$$
f_{x}:[0,1] \rightarrow[0,1]
$$

Definition 4. ([19]) Let $a \in[0,1]$. The characteristic function of $a$ is $\bar{a}:[0,1] \rightarrow[0,1]$, where

$$
\bar{a}(x)= \begin{cases}1 & \text { if } x=a \\ 0 & \text { if } x \neq a\end{cases}
$$

Let $\mathbf{J} \subset \mathbf{M}$ be the set of all characteristic functions of the elements of $[0,1]$, that is, $\mathbf{J}=\{\bar{a}:[0,1] \rightarrow[0,1]: a \in$ $[0,1]\}$. There is a bijection from $\mathbf{J}$ to $[0,1]$, set of membership values of the fuzzy sets.

Definition 5. ([19]) Let $[a, b] \subseteq[0,1]$. The characteristic function of $[a, b]$ is $\overline{[a, b]}:[0,1] \rightarrow[0,1]$, where

$$
\overline{[a, b]}(x)= \begin{cases}1 & \text { if } x \in[a, b] \\ 0 & \text { if } x \notin[a, b]\end{cases}
$$

Let $\mathbf{K} \subset \mathbf{M}$ be the set of all characteristic functions of the closed subintervals of $[0,1]$. There is a bijection from $\mathbf{K}$ to the set $I([0,1])$ of membership values of the IVFSs. An interval type-2 fuzzy set (IT2FS) is a T2FS where all the membership degrees are functions in $\mathbf{K}$. The notation between two slashes, for example $/ a, b /$, refers to an general (closed or unclosed) interval in $[0,1]$, and its characteristic function is $\overline{/ a, b /}$, defined in a similar fashion to Definition 5. Note that the support of function $\overline{\mid a, b /}$ is $\mid a, b /$, and could be an empty set if $a=b$ and is unclosed, in which case $\overline{\mid a, b /}=0$ (where 0 is the constant function $0(x)=0$, for all $x \in[0,1])$.

Walker and Walker justify in [19] that the operations on $\operatorname{Map}(X, \mathbf{M})$ can be defined naturally from the operations on $\mathbf{M}$ and have the same properties. In fact, given the operation $*: \mathbf{M} \times \mathbf{M} \rightarrow \mathbf{M}$, we can define the operation $\star: \operatorname{Map}(X, \mathbf{M}) \times \operatorname{Map}(X, \mathbf{M}) \rightarrow \operatorname{Map}(X, \mathbf{M})$, such that, for each pair $f, g \in \operatorname{Map}(X, \mathbf{M})$, we have $(f \star g)(x)=f(x) * g(x)$, for all $\mathbf{x}$, where $f(x), g(x) \in \mathbf{M}$ (see [19], [10]). Therefore, in this paper, we will work on $\mathbf{M}$, as all the results are easily and directly extensible to $\operatorname{Map}(X, \mathbf{M})$.

Definition 6. ([7], [19], [4], [5]) The operations of $\sqcup$ (generalized maximum), $\sqcap$ (generalized minimum), $\neg$ and the elements $\overline{0}$ y $\overline{1}$ are defined on $\mathbf{M}$ as follows:

$$
(f \sqcup g)(x)=\sup \{f(y) \wedge g(z): y \vee z=x\},
$$

$$
\begin{gathered}
(f \sqcap g)(x)=\sup \{f(y) \wedge g(z): y \wedge z=x\}, \\
\neg f(x)=\sup \{f(y): 1-y=x\}=f(1-x), \\
\overline{0}(x)=\left\{\begin{array}{ll}
1 & \text { if } x=0 \\
0 & \text { if } x \neq 0
\end{array}, \quad \overline{1}(x)=\left\{\begin{array}{ll}
1 & \text { if } x=1 \\
0 & \text { if } x \neq 1 .
\end{array},\right.\right.
\end{gathered}
$$

where $\vee$ and $\wedge$ are the maximum and minimum operations, respectively, on lattice $[0,1]$. Note that $\overline{0}$ and $\overline{1}$ are precisely the characteristic functions of 0 and 1 , respectively.

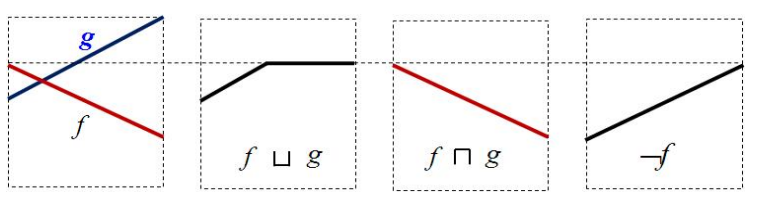

Figure 1: Example of the operations of $\sqcup, \sqcap$, and $\neg$.

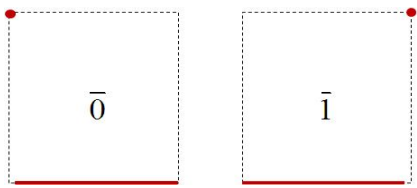

Figure 2: Functions $\overline{0}$ and $\overline{1}$.

We can easily prove that $\sqcup$ and $\sqcap$ satisfy De Morgan's laws with respect to the given operation $\neg$, but $\mathbb{M}=(\mathbf{M}, \sqcup, \sqcap, \neg, \overline{0}, \overline{1})$ does not have a lattice structure, as it does not comply with the absorption law [7], [19]. On the other hand, the operations $\sqcup$ and $\sqcap$ have the properties required to each define a partial order on $\mathbf{M}$.

Definition 7. ([15], [19]) The partial orders defined on $\mathbf{M}$ are as follows:

$$
f \sqsubseteq g \text { if } f \sqcap g=f ; f \preceq g \text { if } f \sqcup g=g .
$$

These two partial orders do not generally coincide [15, 19].

The following definition and theorem were given in previous papers in order to facilitate operations on $\mathbf{M}$ :

Definition 8. ([7], [19], [4], [5]) If $f \in \mathbf{M}, f^{L}, f^{R} \in \mathbf{M}$ are defined as

$$
\begin{aligned}
& f^{L}(x)=\sup \{f(y): y \leq x\}, \\
& f^{R}(x)=\sup \{f(y): y \geq x\} .
\end{aligned}
$$
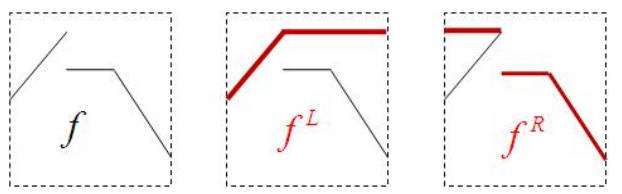

Figure 3: Examples of $f^{L}$ and $f^{R}$.

$f^{L}$ and $f^{R}$ are monotonically increasing and decreasing, respectively. Note that, $f \leq f^{L}, f \leq f^{R},\left(f^{L}\right)^{L}=f^{L}$, $\left(f^{R}\right)^{R}=f^{R}$, and $\left(f^{L}\right)^{R}=\left(f^{R}\right)^{L}=\sup f$, for all $f, g \in \mathbf{M}$ ([19]), where $\leq$ is the usual order in the set of functions ( $f \leq g$ if and only if $f(x) \leq g(x), \forall x$ ). 
Theorem 1. ([19]) Let $f, g \in \mathbf{M}$. Then :

$$
\begin{aligned}
& f \sqsubseteq g \Leftrightarrow\left(f^{R} \wedge g\right) \leq f \leq g^{R}, \\
& f \preceq g \Leftrightarrow\left(g^{L} \wedge f\right) \leq g \leq f^{L} .
\end{aligned}
$$

In the following, we will consider $\mathbf{L}$, the subset of normal and convex functions of $\mathbf{M}$. This set will contain a bounded and complete lattice structure, thanks to which aggregation operators can be properly constructed.

Definition 9. A function $f \in \mathbf{M}$ is convex if, for any $x \leq y \leq z$, we have that $f(y) \geq f(x) \wedge f(z)$

Let $\mathbf{C}$ denote the set of all convex functions in $\mathbf{M}$

Definition 10. ([6], [7], [19], [10]) A function $f \in \mathbf{M}$ is normal (strongly normal) if $\sup \{f(x): x \in[0,1]\}=1$ ( $\mathrm{f}(\mathrm{x})=1$ for some $x \in[0,1])$.

Let $\mathbf{N}$ denote the set of all normal functions in $\mathbf{M}$, and $\mathbf{N}^{*}$ the set of all strongly normal functions in $\mathbf{M}$. It is $\mathbf{N}^{*} \subset \mathbf{N}$ but $\mathbf{N}^{*} \neq \mathbf{N}$. Note that given $f \in \mathbf{M}$, we have that $f \in \mathbf{N}$ if and only if $f^{L} \vee f^{R}=1$, where 1 is the function such that $1(x)=1$, for all $x \in[0,1]$. The equation $f^{L} \vee f^{R}=1$ is equivalent to any of the following four properties: a) $f^{L}(x)=1$ or $f^{R}(x)=1$, for all $x \in[0,1]$, b) $f^{R}(0)=1$, c) $f^{L}(1)=1$, d) $\left(f^{L}\right)^{R}=\left(f^{R}\right)^{L}=1$.

The set of all normal and convex functions of $\mathbf{M}$ will be denoted by $\mathbf{L}$, and the set of all strongly normal and convex functions by $\mathbf{L}^{*}$. The algebra $\mathbb{L}=$ $(\mathbf{L}, \sqcup, \sqcap, \neg, \overline{0}, \overline{1})$ is a subalgebra of $\mathbb{M}$. The partial orders $\sqsubseteq$ and $\preceq$ on $\mathbf{L}$ coincide, and $\mathbb{L}$ is a bounded complete lattice $(\overline{0}$ and $\overline{1}$ are the minimum and maximum, respectively) (see [6], [7], [15], [19]). Besides, it is obvious that $\mathbf{J} \subset \mathbf{K} \subset \mathbf{L}^{*} \subset \mathbf{L} \subset \mathbf{N} \subset \mathbf{M}$. There exists an order isomorphism from $(\mathbf{J}, \sqsubseteq)$ to the interval $([0,1], \leq)$. There also exists an order isomorphism from $(\mathbf{K}, \sqsubseteq)$ to the set $\left(I([0,1]), \leq_{I}\right)$.

The following characterization will be useful for establishing new results:

Theorem 2. ([6], [7]) Let $f, g \in \mathbf{L} . f \sqsubseteq g$ if and only if

$$
g^{L} \leq f^{L} \text { and } f^{R} \leq g^{R} .
$$
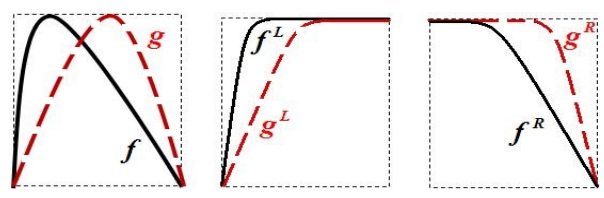

Figure 4: Example where $f \sqsubseteq g$.

\section{2. $\mathrm{N}$-ary aggregation operators}

Remember that:

Definition 11. ([13, 16, 17]) A function $A:[0,1]^{n} \rightarrow$ $[0,1]$ is an $n$-ary aggregation operator on $[0,1]$ if and only if : i) $A(0, \ldots, 0)=0$,

ii) $A(1, \ldots, 1)=1$,

iii) If $x_{i} \leq y_{i}$, for all $i=1, \ldots, n$, then $A\left(x_{1}, \ldots, x_{n}\right) \leq$ $A\left(y_{1}, \ldots, y_{n}\right)$ (increasing in each argument), for all $x_{i}, y_{i} \in[0,1]$.

Any t-norm (triangular norm) and t-conorm on $[0,1]$ is an aggregation function on $[0,1]$. The arithmetic mean $A\left(x_{1}, \ldots, x_{n}\right)=\sum_{i=1}^{n} x_{i} / n$, for all $x_{1}, \ldots, x_{n} \in[0,1]$, also is.

According to the Zadeh's extension principle [24], Z. Takáč $[16,17]$ extended the $n$-ary aggregation operator on $[0,1]$ (see Definition 11 ) to the following $n$-ary operator on $\mathbf{M}$.

Definition 12. ([16, 17]) Let $A:[0,1]^{n} \rightarrow[0,1]$ be an aggregation $n$-ary operator on $[0,1]$. The $n$-ary operator on $\mathbf{M}, \tilde{A}: \mathbf{M}^{n} \rightarrow \mathbf{M}$, is given by

$$
\begin{gathered}
\tilde{A}\left(f_{1}, \ldots, f_{n}\right)(x)= \\
\sup \left\{f_{1}\left(y_{1}\right) \wedge \ldots \wedge f_{n}\left(y_{n}\right): A\left(y_{1}, \ldots, y_{n}\right)=x\right\},
\end{gathered}
$$

where $x, y_{1}, \ldots, y_{n} \in[0,1]$ and $f_{1}, \ldots, f_{n} \in \mathbf{M}$.

Nevertheless, note that in order the operator $\tilde{A}$ be properly defined for all $x \in[0,1]$, it is necessary to demand that the images of the function $A$ contains all values of the interval $[0,1]$, that is, $A$ must be surjective. For example, as the type- 1 operator

$$
A\left(y_{1}, \ldots, y_{n}\right)=\left\{\begin{array}{cc}
1 & \text { if } y_{1}=\ldots=y_{n}=1, \\
0 & \text { otherwise }
\end{array}\right.
$$

is not surjective, $\tilde{A}$ is not defined for all $x \in[0,1]$ ( $\tilde{A}$ is not defined for $x \in(0,1)$ ).

Furthermore, Z. Takáč introduced, in the same papers the type-2 aggregation operators.

Definition 13. ([16]) Let $(\mathbf{U}, \sqcup, \sqcap, \overline{0}, \overline{1}, \sqsubseteq, \preceq)$ be a subalgebra of $(\mathbf{M}, \sqcup, \sqcap, \overline{0}, \overline{1}, \sqsubseteq, \preceq)$. A function $\chi: \mathbf{U}^{n} \rightarrow \mathbf{U}$ is a type-2 aggregation operator on $\mathbf{U}$ if and only if the following conditions 1),2) and one of the conditions 3 ) and $\left.3^{\prime}\right)$ are fullfiled:

1) $\chi(\overline{0}, \ldots, \overline{0})=\overline{0}$

2) $\chi(\overline{1}, \ldots, \overline{1})=\overline{1}$

3) If $f_{i} \sqsubseteq g_{i}$, for all $i=1, \ldots, n$, then $\chi\left(f_{1}, \ldots, f_{n}\right) \sqsubseteq$ $\chi\left(g_{1}, \ldots, g_{n}\right)$ (increasing in each argument), for all $f_{i}, g_{i} \in \mathbf{U}$.

3') If $f_{i} \preceq g_{i}$, for all $i=1, \ldots, n$, then $\chi\left(f_{1}, \ldots, f_{n}\right) \preceq$ $\chi\left(g_{1}, \ldots, g_{n}\right) \quad$ (increasing in each argument), for all $f_{i}, g_{i} \in \mathbf{U}$.

Remember that in $\mathbf{L} ， \sqsubseteq \equiv \preceq$, and so the conditions 3 ) and 3') are the same.

Z. Takáč $[16,17]$ also determined that if $A$ is a continuous $n$-ary aggregation operator on $[0,1]$, then $\tilde{A}$ is an aggregation operator on $\mathbf{L}^{*}$ (that is a subset of $\mathbf{L}$ ). Nevertheless he did not give any aggregation operator on $\mathbf{L}$, or on $\mathbf{M}$, as the closure properties given in those papers were established only on $\mathbf{L}^{*}$.

Now we propose the definition of $n$-ary aggregation operator on a bounded and partially ordered set. This definition is more general than that give in Definition 13 and will allow us to properly obtain aggregation operators on $\mathbf{M}$, and on any bounded subset of $\mathbf{M}$. 
Definition 14. Let $U$ be a set and $\leq_{U}$ be a partial order in $U$ such that $\left(U, \leq_{U}\right)$ has a minimum element $0_{\leq_{U}}$ and a maximum element $1_{\leq_{U}}$. An $n$-ary aggregation operator on $\left(U, \leq_{U}\right)$ is a function $\chi: U^{n} \rightarrow U$ such that:

1) $\chi\left(0_{\leq_{U}}, \ldots, 0_{\leq_{U}}\right)=0_{\leq_{U}}$,

2) $\chi\left(1_{\leq_{U}}, \ldots, 1_{\leq_{U}}\right)=1_{\leq_{U}}$,

3) Given $f_{i}, g_{i} \in U$, if $f_{i} \leq_{U} g_{i}$, for all $i=1, \ldots, n$, then $\chi\left(f_{1}, \ldots, f_{n}\right) \leq_{U} \chi\left(g_{1}, \ldots, g_{n}\right)$ (increasing in each argument).

Similarly to Definition 13, an $n$-ary aggregation operator in $(\mathbf{L}, \sqsubseteq)$ will be called a type-2 aggregation operator on $\mathbf{L}$.

\section{Aggregation operators from Zadeh's extension principle}

In this Section we define, from the Zadeh's extension principle, new $n$-operators on $\mathbf{M}$, more general than those given by Z. Takáć [16] (see Definition 12). We also determine some of their general properties.

Definition 15. Let $\phi:[0,1]^{n} \rightarrow[0,1]$ a surjective $n$-ary operator on $[0,1]$, and $\star:[0,1]^{n} \rightarrow[0,1]$ be an $n$-ary operator on $[0,1]$. The $n$-ary operator on $\mathbf{M}, \wedge_{\star, \phi}: \mathbf{M}^{n} \rightarrow \mathbf{M}$, is defined as

$$
\begin{gathered}
\curlywedge_{\star, \phi}\left(f_{1}, \ldots, f_{n}\right)(x)= \\
\sup \left\{\star\left(f_{1}\left(y_{1}\right), \ldots, f_{n}\left(y_{n}\right)\right): \phi\left(y_{1}, \ldots, y_{n}\right)=x\right\},
\end{gathered}
$$

where $x, y_{1}, \ldots, y_{n} \in[0,1]$ and $f_{1}, \ldots, f_{n} \in \mathbf{M}$.

Note that if $\star\left(f_{1}\left(y_{1}\right), \ldots, f_{n}\left(y_{n}\right)\right)=f_{1}\left(y_{1}\right) \wedge f_{2}\left(y_{2}\right) \wedge \ldots \wedge$ $f_{n}\left(y_{n}\right)$, and $\phi$ is a continuous $n$-ary aggregation operator on $[0,1]$, then, according to $[16,17], \wedge_{\star, \phi}$ is an $n$-ary aggregation operator on $\mathbf{L}^{*}$.

Example 1. If $\star\left(z_{1}, z_{2}, z_{3}\right)=\left(z_{1} \vee z_{2}\right) \wedge z_{3}$ for all $z_{1}, z_{2}$, $z_{3} \in[0,1]$, and $\phi$ is the arithmetic mean, then we have for all $f_{1}, f_{2}, f_{3} \in \mathbf{M}$,

$$
\begin{gathered}
\wedge_{\star, \phi}\left(f_{1}, f_{2}, f_{3}\right)(x)= \\
\sup \left\{\left(f_{1}\left(y_{1}\right) \vee f_{2}\left(y_{2}\right)\right) \wedge f_{3}\left(y_{3}\right): \frac{y_{1}+y_{2}+y_{3}}{3}=x\right\} .
\end{gathered}
$$

$\widehat{\star}_{\star, \phi}$ is not an aggregation operator, as it does not satisfy the boundary conditions. In fact,

$$
ᄉ_{\star, \phi}(\overline{0}, \overline{0}, \overline{0}) \neq \overline{0},
$$

for example, $\wedge_{\star, \phi}(\overline{0}, \overline{0}, \overline{0})\left(\frac{1}{3}\right)=1$. And also

$$
\curlywedge_{\star, \phi}(\overline{1}, \overline{1}, \overline{1}) \neq \overline{1} .
$$

This is because 0 is not an absorbent element of the operator $\star$.

The operator $\lambda_{\star, \phi}$ of Definition 15, is also a generalization of the binary operations given by Hernández and al. in $[8,9]$. There $\star$ was any binary operation on $[0,1]$, and $\phi$ a t-norm (or t-conorm) in [0,1]. In [8] it was proved that if $\star$ is any continuous t-norm in $[0,1]$, and $\phi$ is any continuous t-norm (t-conorm) in $[0,1]$, then $\curlywedge_{\star, \phi}$ is a $\mathrm{t}_{r}$-norm ( $\mathrm{t}_{r}$-conorm) in $\mathbf{L}\left(\mathrm{t}_{r}\right.$-norm or $\mathrm{t}_{r}$-conorm is a t-norm or t-conorm according to the restrictive axioms, see [20]). Nevertheless in this work $\phi$ is any surjective $n$-ary operator on $[0,1]$, and the main goal will be to obtain $n$-ary aggregation operators on $\mathbf{L}$. For this purpose, we firstly give some previous results.

From now on $\curlywedge_{\star, \phi}, \phi$ and $\star$ will be the operations introduced in Definition 15, taking into account that $\phi$ will always be surjective.

Proposition 1. If $\star(1, \ldots, 1)=1$ and 0 is an absorbent element of $\star$, then

- If $\phi(0, \ldots, 0)=0$, it is $\curlywedge_{\star, \phi}(\overline{0}, \ldots, \overline{0})=\overline{0}$.

- If $\phi(1, \ldots, 1)=1$, it is $\curlywedge_{\star, \phi}(\overline{1}, \ldots, \overline{1})=\overline{1}$.

\section{Proposition 2.}

- If $\star(1, \ldots, 1)=1$, then $\curlywedge_{\star, \phi}(1, \ldots, 1)=1$.

- If 0 is an absorbent element of $\star$, for any $f_{1}, \ldots, f_{n} \in \mathbf{M}$, such that $f_{i}=0$ (empty function) for some $i=1, \ldots, n$, it is $\curlywedge_{\star, \phi}\left(f_{1}, \ldots, f_{n}\right)=0$ (empty).

Proposition 3. If $\star$ is increasing in each argument, and $f_{1}, \ldots, f_{n}, g_{1}, \ldots, g_{n} \in \mathbf{M}$, such that $f_{1} \leq g_{1}, \ldots, f_{n} \leq g_{n}$, then

$$
\curlywedge_{\star, \phi}\left(f_{1}, \ldots, f_{n}\right) \leq \curlywedge_{\star, \phi}\left(g_{1}, \ldots, g_{n}\right) .
$$

Proposition 4. If both $\phi$ and $\star$ are continuous and increasing in each argument, and $f_{1}, \ldots, f_{n} \in \mathbf{M}$, it is

$$
\begin{gathered}
\left(\curlywedge_{\star, \phi}\left(f_{1}, \ldots, f_{n}\right)\right)^{L}=\curlywedge_{\star, \phi}\left(f_{1}^{L}, \ldots, f_{n}^{L}\right), \\
\left(\curlywedge^{L}\left(f_{1}, \ldots, f_{n}\right)\right)^{R}=\curlywedge_{\star, \phi}\left(f_{1}^{R}, \ldots, f_{n}^{R}\right) .
\end{gathered}
$$

Remark 1. There exist cases in which $\star$ is not continuous, and although the rest of conditions of this Proposition 4 are fulfilled, the equalities do not hold. For example, let us consider

$$
\begin{gathered}
\star(u, v)=\left\{\begin{array}{lc}
u, & \text { if } v=1, \\
v, & \text { if } u=1, \\
0, & \text { otherwise, }
\end{array}\right. \\
f(x)=\left\{\begin{array}{cc}
x, & \text { if } x \in[0,1), \\
0.3, & \text { otherwise, }
\end{array}\right.
\end{gathered}
$$

and $\phi$ any continuous binary aggregation operator on [0,1]. We have $f(x)<1, \quad$ for all $x \in[0,1]$, and sup $f=1$. Then, $(\curlywedge(f, f))^{R}(0)=$ $\sup \left\{\star\left(f\left(y_{1}\right), f\left(y_{2}\right)\right): \phi\left(y_{1}, y_{2}\right) \geq 0\right\}=\sup \{0\}=0$, and $\wedge_{\star, \phi}\left(f^{R}, f^{R}\right)(0)=\sup \left\{\star\left(f^{R}\left(y_{1}\right), f^{R}\left(y_{2}\right)\right)\right.$ : $\left.\phi\left(y_{1}, y_{2}\right)=0\right\}=\star\left(f^{R}(0), f^{R}(0)\right)=\star(1,1)=1$. So, $\left(\wedge_{\star, \phi}(f, f)\right)^{R}(0) \neq \Lambda_{\star, \phi}\left(f^{R}, f^{R}\right)(0)$.

On the other hand, $\left(\Lambda_{\star, \phi}(f, f)\right)^{L}(1)=$ $\sup \left\{\star\left(f\left(y_{1}\right), f\left(y_{2}\right)\right): \phi\left(y_{1}, y_{2}\right) \leq 1\right\}=\sup \{0\}=0$. And $ᄉ_{\star, \phi}\left(f^{L}, f^{L}\right)(1)=\sup \left\{\star\left(f^{L}\left(y_{1}\right), f^{L}\left(y_{2}\right)\right)\right.$ : $\left.\phi\left(y_{1}, y_{2}\right)=1\right\}=\star\left(f^{L}(1), f^{L}(1)\right)=1$. Therefore, $\left(\curlywedge_{\star, \phi}(f, f)\right)^{L}(1) \neq \wedge_{\star, \phi}\left(f^{L}, f^{L}\right)(1)$. L.

Let us now see the closure properties in $\mathbf{N}, \mathbf{K}, \mathbf{J}, \mathbf{C}$ y

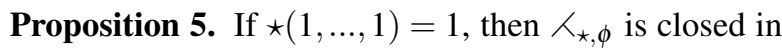
N*. 
Proposition 6. Let $\star$ be increasing in each argument, continuous at $(1, \ldots, 1) \in[0,1]^{n}$, and such that $\star(1, \ldots, 1)=1$. Furthermore, let $\phi$ be continuous and increasing in each argument. Then $\widehat{ }_{\star, \phi}$ is closed on $\mathbf{N}$.

Remark 2. The example in the Remark 1 also proves that there exist cases in which $\star$ is not continuous at the point $(1, \ldots, 1)$, and although the rest of the conditions required in this Proposition 6 are satisfied, $\wedge_{\star, \phi}$ is closed neither on $\mathbf{N}$, nor on $\mathbf{L}$. In fact, in this case, $f \in \mathbf{N}$, $f \in \mathbf{L}$, and $\left(\curlywedge_{\star, \phi}(f, f)\right)^{R}(0)=0 \neq 1$, so $\curlywedge_{\star, \phi}(f, f)$ is not normal, and it is not in $\mathbf{L}$.

On the other hand, examples of binary operations $\star$ satisfying the required conditions in this Proposition 6 are the t-norms and t-conorms continuous at $(1,1)$ and the operation

$$
\star(u, v)=\left\{\begin{array}{cc}
u \wedge v, & \text { if } u \geq 0.8 \text { and } v \geq 0.8, \\
0, & \text { otherwise. }
\end{array}\right.
$$

Proposition 7. If $\phi$ is continuous and increasing in each argument, $\star(1, \ldots, 1)=1$ and 0 is the absorbent element of $\star$, then for all collection of elements $\left\{\overline{\left[a_{i}, b_{i}\right]} \in \mathbf{K}\right\}$, we have

$\curlywedge_{\star, \phi}\left(\overline{\left[a_{1}, b_{1}\right]}, \ldots, \overline{\left[a_{n}, b_{n}\right]}\right)=\overline{\left[\phi\left(a_{1}, \ldots, a_{n}\right), \phi\left(b_{n}, \ldots, b_{n}\right)\right]}$,

where $\left[\phi\left(a_{1}, \ldots, a_{n}\right), \phi\left(b_{n}, \ldots, b_{n}\right)\right] \in I([0,1])$. That is, $\Lambda_{\star, \phi}$ is closed on $\mathbf{K}$.

Remark 3. There are cases in which $\phi$ is not continuous, although all other conditions in Proposition 7 are fulfilled, and $\lambda_{\star, \phi}$ is not closed on $\mathbf{K}$. For example, let $\star$ any t-norm. And let us consider the noncontinuous t-norm $\phi$ which was given in Remark 1 . Then, $\phi(\overline{[0,1]}, \overline{[0.3,0.7]})=g$, where $g \in \mathbf{M}$ is

$$
g(x)=\left\{\begin{array}{lc}
1, & \text { if } x=0 \text { or } x \in[0.3,0.7] \\
0, & \text { otherwise }
\end{array}\right.
$$

Note that $g \notin \mathbf{K}$.

Corollary 1. Let $\bar{a}_{i} \in \mathbf{J}$, for all $i=1, \ldots, n$. Under the same conditions as in Proposition 7, we have

$\aleph_{\star, \phi}\left(\overline{a_{1}}, \ldots, \overline{a_{n}}\right)=\bar{e}, \quad$ where $e=\phi\left(a_{1}, \ldots, a_{n}\right) \in[0,1]$.

That is, $\wedge_{\star, \phi}$ is closed on $\mathbf{J}$

Corollary 2. Let $\phi$ be a continuous binary operator, increasing in each argument, with neutral and absorbent elements 1 and 0 , respectively, and let $\star$ be a binary operator, with $\star(1,1)=1$ and absorbent element 0 . Then

$$
\wedge_{\star, \phi}(\overline{[0,1]}, \overline{[a, b]})=\overline{[0, b]} .
$$

Remark 4. There are cases in which $\phi$ is not continuous, although all other conditions in Corollary 2 are fulfilled, and the conclusion in that proposition is not satisfied (see Remark 3).

Corollary 3. Let $\phi$ binary, continuous, increasing in each argument, with 0 and 1 as neutral and absorbent elements, respectively, and let $\star$ binary, with $\star(1,1)=1$ and absorbent element 0 . Then

$$
\wedge_{\star, \phi}(\overline{[0,1]}, \overline{[a, b]})=\overline{[a, 1]} .
$$

Let us now see the case in which the arguments of the operator $\lambda_{\star, \phi}$ are general (not necessarily closed) intervals.

Proposition 8. Let $\overline{a_{i}, b_{i} /}$ be, for all $i=1, \ldots, n$, the characteristic function of an interval. Under the same conditions as in the Proposition 7, we have

If $\overline{\left|a_{i}, b_{i}\right|}=0 \quad$ (empty set), for some $i$, then $<\left(\overline{/ a_{1}, b_{1} /}, \ldots, \overline{/ a_{n}, b_{n} /}\right)=0$.

If $\overline{/ a_{i}, b_{i} /} \neq 0$, for all $i$, then $<\left(\overline{\left.\mid a_{1}, b_{1} /, \ldots, \overline{\left|a_{n}, b_{n}\right|}\right)}\right.$ $=\overline{/ \phi\left(a_{1}, \ldots, a_{n}\right), \phi\left(b_{1}, \ldots, b_{n}\right) /} \neq 0$ (non-empty).

Proposition 9. Let $\phi$ continuous and increasing in each argument, and $\star$ increasing in each argument, with absorbent element 0 , and $\star(1, \ldots, 1)=1$, then $\wedge_{\star, \phi}$ is closed on $\mathbf{L}^{*}$.

If, in addition, $\star$ is continuous at the point $(1, \ldots, 1)$, $\Lambda_{\star, \phi}$ is closed on $\mathbf{L}$.

Remark 5. There are cases in which $\star$ is not continuous at $(1, \ldots, 1)$, although all other conditions in Proposition 9 are fulfilled, and ${{ }_{\star}, \phi}_{\phi}$ is not closed on L. See Remark 2.

Also, there are cases in which $\phi$ is not continuous, although all other conditions in Proposition 9 are fulfilled, and $\lambda_{\star, \phi}$ is closed neither on $\mathbf{L}$ nor on $\mathbf{L}^{*}$. See the example in Remark 3.

Let study the increase in each argument of the operator $\Lambda_{\star, \phi}$, with respect to the partial order of $\mathbf{L}$.

Proposition 10. Under the same conditions as in the Proposition 9, $\wedge_{\star, \phi}$ is increasing in each argument on $\mathbf{L}^{*}$, respect to the partial order of $\mathbf{L}$.

In addition, if $\star$ is continuous at the point $(1, \ldots, 1) \in$ $[0,1]^{n}, \wedge_{\star, \phi}$ is increasing in each argument on $\mathbf{L}$, respect to the partial order of $\mathbf{L}$.

From the previous results, it is direct to obtain the following Proposition.

Proposition 11. Let $\phi$ be a continuous $n$-ary aggregation operator on $[0,1]$. And $\star$ an $n$-ary operator with absorbent element 0 , increasing in each argument and such that $\star(1, \ldots, 1)=1$. Then $\curlywedge_{\star, \phi}$ is an $n$-ary aggregation operator on $\mathbf{L}^{*}$.

If, in addition, $\star$ is continuous at the point $(1, \ldots, 1) \in[0,1]^{n}$, then $\lambda_{\star, \phi}$ is an $n$-ary aggregation operator on $\mathbf{L}$.

Example 2. i) If $\phi$ is any binary and continuous aggregation operator on $[0,1]$ and $\star$ is a t-norm on $[0,1]$, con-

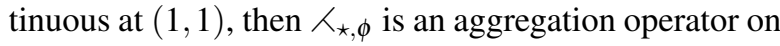
L.

ii) If $\phi$ is any binary and continuous aggregation operator on $[0,1]$, and $\star$ is the binary operation, which is not a t-norm:

$$
\star(u, v)=\left\{\begin{array}{cc}
u . v, & \text { if } u \geq 0.9 \text { and } v \geq 0.9, \\
0, & \text { otherwise. }
\end{array}\right.
$$

then $\widehat{ }_{\star, \phi}$ is a binary aggregation operator on $\mathbf{L}$. 
iii) If $\phi$ is any continuous binary aggregation operator on $[0,1]$ and $\star$ is a t-norm on $[0,1]$, then $\widehat{K}_{\star, \phi}$ is a binary aggregation operator on $\mathbf{L}^{*}$.

iv) If $\phi$ is any binary and continuous aggregation operator on $[0,1]$, and $\star$ is the binary operation, which is not a t-norm:

$$
\star(u, v)=\left\{\begin{array}{lc}
1, & \text { if } u=1 \text { and } v=1, \\
0, & \text { otherwise }
\end{array}\right.
$$

then $\curlywedge_{\star, \phi}$ is a binary aggregation operator on $\mathbf{L}^{*}$.

\section{Other aggregation operators on $M$}

In this section we present other operators different to those obtained from the Zadeh's extension principle, and study the conditions under which they are aggregation operators.

Definition 16. Let $\phi:[0,1]^{n} \rightarrow[0,1]$, an $n$-ary aggregation operator on $[0,1]$. We define the $n$-ary operators on $\mathbf{M}, \chi_{\phi}^{L}: \mathbf{M}^{n} \rightarrow \mathbf{M}$ and $\chi_{\phi}^{R}: \mathbf{M}^{n} \rightarrow \mathbf{M}$, as

$\chi_{\phi}^{L}\left(f_{1}, \ldots, f_{n}\right)(x)=\left\{\begin{array}{cl}\overline{0}(x) & \text { if } f_{i}=\overline{0} \forall i, \\ \phi\left(f_{1}^{L}(x), \ldots, f_{n}^{L}(x)\right) & \text { otherwise. }\end{array}\right.$,

$\chi_{\phi}^{R}\left(f_{1}, \ldots, f_{n}\right)(x)=\left\{\begin{array}{cl}\overline{1}(x) & \text { if } f_{i}=\overline{1} \forall i \\ \phi\left(f_{1}^{R}(x), \ldots, f_{n}^{R}(x)\right) & \text { otherwise. }\end{array}\right.$,

where $x \in[0,1]$ and $f_{1}, \ldots, f_{n} \in \mathbf{M}$.

Proposition 12. $\chi_{\phi}^{L}$ is an aggregation operator on $(\mathbf{M}$, $\preceq)$, and $\chi_{\phi}^{R}$ is an aggregation operator on (M,

Proof. Remember that $\overline{0}$ and $0(0(x)=0$, for all $x)$ are, respectively, the minimun and maximum of $(\mathbf{M}, \preceq)$. Also, 0 and $\overline{1}$ are, respectively, the minimun and maximum of the poset $(\mathbf{M}, \sqsubseteq)$.

Let us prove that $\chi_{\phi}^{L}$ is an aggregation operator on $(\mathbf{M}$, $\preceq$ ), and $\chi_{\phi}^{R}$ is an aggregation operator on (M, ing to Definition 14 .

Firstly, the boundary conditions are fulfilled, that is :

$$
\begin{aligned}
& \chi_{\phi}^{L}(\overline{0}, \ldots, \overline{0})=\overline{0}, \\
& \chi_{\phi}^{L}(0, \ldots, 0)=0, \\
& \chi_{\phi}^{R}(0, \ldots, 0)=0, \\
& \chi_{\phi}^{R}(\overline{1}, \ldots, \overline{1})=\overline{1} .
\end{aligned}
$$

We now study the increase in each argument of $\chi_{\phi}^{L}$ in the poset (M, $\preceq$ ). We should prove that if $f_{i} \preceq g_{i}$, then

$$
\chi_{\phi}^{L}\left(f_{1}, \ldots, f_{n}\right) \preceq \chi_{\phi}^{L}\left(g_{1}, \ldots, g_{n}\right),
$$

that is, according to Theorem 1 ,

$$
\begin{gathered}
\left(\chi_{\phi}^{L}\left(g_{1}, \ldots, g_{n}\right)(x)\right)^{L} \wedge \chi_{\phi}^{L}\left(f_{1}, \ldots, f_{n}\right)(x) \\
\leq \chi_{\phi}^{L}\left(g_{1}, \ldots, g_{n}\right)(x) \leq\left(\chi_{\phi}^{L}\left(f_{1}, \ldots, f_{n}\right)(x)\right)^{L} \text {, for all } x .
\end{gathered}
$$

Two cases can hold: i) $f_{i}=\overline{0} \forall i$. In this case, $\chi_{\phi}^{L}\left(f_{1}, \ldots, f_{n}\right)=\overline{0} \preceq \chi_{\phi}^{L}\left(g_{1}, \ldots, g_{n}\right)$, as $\overline{0}$ is the minimum in $(\mathbf{M}, \preceq)$. ii) $f_{j} \neq \overline{0}$, for some $j$, and so $g_{j} \neq \overline{0}$ for this $j$. In this case, $\chi_{\phi}^{L}\left(f_{1}, \ldots, f_{n}\right)(x)=\phi\left(f_{1}^{L}(x), \ldots, f_{n}^{L}(x)\right)$, and $\chi_{\phi}^{L}\left(g_{1}, \ldots, g_{n}\right)(x)=\phi\left(g_{1}^{L}(x), \ldots, g_{n}^{L}(x)\right)$. We have to prove that

$$
\left(\phi\left(g_{1}^{L}(x), \ldots, g_{n}^{L}(x)\right)\right)^{L} \wedge \phi\left(f_{1}^{L}(x), \ldots, f_{n}^{L}(x)\right)
$$

$\leq \phi\left(g_{1}^{L}(x), \ldots, g_{n}^{L}(x)\right) \leq\left(\phi\left(f_{1}^{L}(x), \ldots, f_{n}^{L}(x)\right)\right)^{L}$, for all $x$. $\phi\left(f_{1}^{L}(x), \ldots, f_{n}^{L}(x)\right)$ and $\phi\left(g_{1}^{L}(x), \ldots, g_{n}^{L}(x)\right)$ are increasing, and so $\left(\phi\left(f_{1}^{L}(x), \ldots, f_{n}^{L}(x)\right)\right)^{L}=\phi\left(f_{1}^{L}(x), \ldots, f_{n}^{L}(x)\right)$ and $\left(\phi\left(g_{1}^{L}(x), \ldots, g_{n}^{L}(x)\right)\right)^{L}=\phi\left(g_{1}^{L}(x), \ldots, g_{n}^{L}(x)\right)$.

As $f_{i} \preceq g_{i}$, newly by Theorem 1 , it is $\left(f_{i} \wedge g_{i}^{L}\right) \leq g_{i} \leq f_{i}^{L}$. Then we deduce that $g_{i}^{L} \leq\left(f_{i}^{L}\right)^{L}=f_{i}^{L}$, and as $\phi$ is increasing in each argument, we obtain $\phi\left(g_{1}^{L}(x), \ldots, g_{n}^{L}(x)\right) \wedge \phi\left(f_{1}^{L}(x), \ldots, f_{n}^{L}(x)\right) \leq$ $\phi\left(g_{1}^{L}(x), \ldots, g_{n}^{L}(x)\right) \leq \phi\left(f_{1}^{L}(x), \ldots, f_{n}^{L}(x)\right)$. Therefore, $\chi_{\phi}^{L}$ is increasing on each argument in the poset (M, $\preceq)$.

Likewise we can obtain that $\chi_{\phi}^{R}$ is increasing on each argument in the poset $(\mathbf{M}, \sqsubseteq)$.

Remark 6. $\chi_{\phi}^{L}$ is not an aggregation operator on $(\mathbf{M}, \sqsubseteq)$, as, in general, is not increasing.

In fact, let us consider the functions in the figure 5 a)

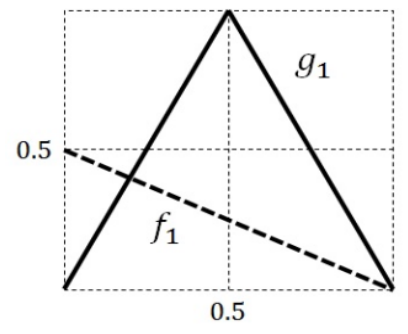

a)

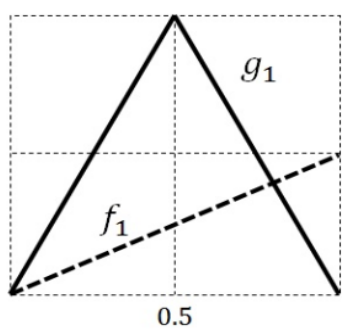

b)
Figure 5: counterexamples for Remarks 6 and 7

and let $f_{2}=g_{2}=\overline{1}$

We have $f_{1} \sqsubseteq g_{1}, \overline{1} \sqsubseteq \overline{1}$. Taking any strictly increasing function $\phi$, we have

$\left(\chi_{\phi}^{L}\left(f_{1}, f_{2}\right)\right)^{R}(0.5)=\phi\left(f_{1}^{L}(1), f_{2}^{L}(1)\right)=\phi(0.5,1)$

$\phi\left(g_{1}^{L}(0.5), g_{2}^{L}(0.5)\right)=\phi(1,0)$

$\phi\left(f_{1}^{L}(0.5), f_{2}^{L}(0.5)\right)=\phi(0.5,0)$

$\phi(0.5,1) \wedge \phi(1,0) \not \varnothing \phi(0.5,0)$, and so

$\left(\chi_{\phi}^{L}\left(f_{1}, f_{2}\right)\right)^{R} \wedge \chi_{\phi}^{L}\left(g_{1}, g_{2}\right) \not \leq \chi_{\phi}^{L}\left(f_{1}, f_{2}\right)$

Remark 7. Furthermore, $\chi_{\phi}^{R}$ is not an aggregation operator on $(\mathbf{M}, \preceq)$.

In fact, let us consider the functions in the figure $5 \mathrm{~b}$ ) and let $f_{2}=g_{2}=\overline{0}$.

We have $g_{1} \preceq f_{1}, \overline{0} \preceq \overline{0}$. Taking any strictly increasing function $\phi$, we have

$\left(\chi_{\phi}^{R}\left(f_{1}, f_{2}\right)\right)^{L}(0.5)=\phi\left(f_{1}^{R}(0), f_{2}^{R}(0)\right)=\phi(0.5,1)$

$\phi\left(g_{1}^{R}(0.5), g_{2}^{R}(0.5)\right)=\phi(1,0)$

$\phi\left(f_{1}^{R}(0.5), f_{2}^{R}(0.5)\right)=\phi(0.5,0)$

$\phi(0.5,1) \wedge \phi(1,0) \$ \phi(0.5,0)$, and so

$\left(\chi_{\phi}^{R}\left(f_{1}, f_{2}\right)\right)^{L} \wedge \chi_{\phi}^{R}\left(g_{1}, g_{2}\right) \nRightarrow \chi_{\phi}^{R}\left(f_{1}, f_{2}\right)$ 
Proposition 13. $\chi_{\phi}^{L}$ and $\chi_{\phi}^{R}$ are closed in $\mathbf{L}$.

Proof. If $f_{i}=\overline{0} \forall i$, it is $\chi_{\phi}^{L}\left(f_{1}, \ldots, f_{n}\right)=\overline{0} \in \mathbf{L}$.

If $f_{i} \neq \overline{0}$, for some $i$, then $\chi_{\phi}^{L}\left(f_{1}, \ldots, f_{n}\right)(x)=$ $\phi\left(f_{1}^{L}(x), \ldots, f_{n}^{L}(x)\right)$.

$\phi\left(f_{1}^{L}(x), \ldots, f_{n}^{L}(x)\right)$ is increasing, and so for all $x \leq y \leq z, \phi\left(f_{1}^{L}(x), \ldots, f_{n}^{L}(x)\right) \leq \phi\left(f_{1}^{L}(y), \ldots, f_{n}^{L}(y)\right) \leq$ $\phi\left(f_{1}^{L}(z), \ldots, f_{n}^{L}(z)\right) . \quad$ Then $\phi\left(f_{1}^{L}(y), \ldots, f_{n}^{L}(y)\right) \geq$ $\phi\left(f_{1}^{L}(x), \ldots, f_{n}^{L}(x)\right) \wedge \phi\left(f_{1}^{L}(z), \ldots, f_{n}^{L}(z)\right), \quad$ and $\chi_{\phi}^{L}$ is convex.

Furthermore, as $f_{i} \in \mathbf{L} \forall i$, we have $f_{i}^{L}(1)=1 \forall i$, and $\chi_{\phi}^{L}\left(f_{1}, \ldots, f_{n}\right)(1)=\phi\left(f_{1}^{L}(1), \ldots, f_{n}^{L}(1)\right)=\phi(1, \ldots, 1)=1$. That is, $\chi_{\phi}^{L}$ is normal, and even strongly normal.

Likewise we can see that $\chi_{\phi}^{R}$ is closed in $\mathbf{L}$.

Proposition 14. $\chi_{\phi}^{L}$ and $\chi_{\phi}^{R}$ are aggregation operators on $(\mathbf{L}, \preceq)$.

Proof. The closure in $\mathbf{L}$ was proved in Proposition 13.

Besides, in $\mathbf{L}$, $\equiv \preceq$, and so, according to Proposition $12, \chi_{\phi}^{L}$ and $\chi_{\phi}^{R}$ are increasing in each argument on $(\mathbf{L}$, $\preceq)$.

Moreover, $\quad \chi_{\phi}^{L}(\overline{1}, \ldots, \overline{1})(x)=\phi\left(\overline{1}^{L}(x), \ldots, \overline{1}^{L}(x)\right)=$ $\phi(\overline{1}(x), \ldots, \overline{1}(x))=\overline{1}(x)$, for all $x$, as $\overline{1}=\overline{1}^{L}$, $\phi(1, \ldots, 1)=1$, and $\phi(0, \ldots, 0)=0$.

Likewise, $\quad \chi_{\phi}^{R}(\overline{0}, \ldots, \overline{0})(x)=\phi\left(\overline{0}^{R}(x), \ldots, \overline{0}^{R}(x)\right)=$ $\phi(\overline{0}(x), \ldots, \overline{0}(x))=\overline{0}(x)$, for all $x$, as $\overline{0}=\overline{0}^{R}$, $\phi(1, \ldots, 1)=1$, and $\phi(0, \ldots, 0)=0$.

Finally, the other two boundary properties were obtained in the proof of the Proposition 12.

Remark 8. These operators $\chi_{\phi}^{L}$ and $\chi_{\phi}^{R}$, are different to the aggregation operators on $\mathbf{L}$, obtained with $\Lambda_{\star}, \phi$, because the later are closed in $\mathbf{J}$, whereas $\chi_{\phi}^{L}$ and $\chi_{\phi}^{R}$ are not.

\section{Conclusions}

In this paper we have defined aggregation operators on any partially ordered and bounded set. Applying the Zadeh's extension principle, we have proposed a family of operators on $(\mathbf{M}, \sqcup, \sqcap, \overline{0}, \overline{1}, \sqsubseteq, \preceq)$ more general than those given by Z. Takáč in [16], and we have studied under which conditions they are aggregation operators on $\mathbf{L}$. Furthermore, we have obtained through a very different way, other aggregation operators on $\mathbf{L}$ and on M.

Future work will focus on obtaining new aggregation operators on $\mathbf{L}$ or on $\mathbf{M}$, by other methods.

Acknowledgements This paper is partially supported by UPM (Spain) and UNET (Venezuela)

\section{References}

[1] H. Bustince, E. Barrenechea and M. Pagola, Generation of interval-valued fuzzy and Atanassov's intuitionistic fuzzy connectives from fuzzy connectives and from $K_{\alpha}$ operators. Laws for conjunctions and disjunctions. Amplitude, International Journal and Intelligence Systems, 23:680-714, Willey, 2008.

[2] G. Deschrijver and E. Kerre, Aggregation operation in interval-valued fuzzy and Atanassov's intuitionistic fuzzy set theory, Fuzzy Sets and Their Extensions: Representation, Aggregation and Models, $\mathrm{H}$. Bustince et al. (Eds.), Springer, Berlin, 183-203, 2008.

[3] D. Dubois and H. Prade, A review of fuzzy set aggregation connectives, Information Sciences, 36, no. 1-2:85-121, Elsevier, 1985.

[4] Z. Gera and J. Dombi, Exact calculations of extended logical operations on fuzzy truth values, Fuzzy Sets and Systems, 159, no. 11:1309-1326, Elsevier, 2008.

[5] Z. Gera and J. Dombi, Type-2 implications on noninteractive fuzzy truth values, Fuzzy Sets and Systems, 159, no. 22:3014-3032, Elsevier, 2008.

[6] J. Harding, C. Walker and E. Walker, Convex normal functions revisited, Fuzzy Sets and Systems, 161:1343-1349, Elsevier, 2010.

[7] J. Harding, C. Walker and E. Walker, Lattices of convex normal functions, Fuzzy Sets and Systems, 159:1061-1071, Elsevier, 2008.

[8] P. Hernández, S. Cubillo and C. TorresBlanc, On t-norms for type-2 fuzzy sets, IEEE Transaction on Fuzzy Systems (2014), http://dx.doi.org/10.1109/TFUZZ.2014.2346247.

[9] P. Hernández, S. Cubillo and C. Torres-Blanc, Negations on type-2 fuzzy sets, Fuzzy Sets and Systems, 252:111-124, Elsevier, 2014.

[10] B. Hu and C. Kwong, On type-2 fuzzy sets and their t-norm operations, Information Sciences, 255:58-81, Elsevier, 2014.

[11] J. Mendel and R. Jhon, Type-2 fuzzy sets made Simple, IEEE Transactions on Fuzzy Systems, 10, no. 2:117-127, IEEE, 2002.

[12] O. Linda and M. Manic, Monotone Centroid Flow Algorithm for Type Reduction of General Type-2 Fuzzy Sets, IEEE Transaction on Fuzzy Systems, 20, no. 5:805-819, IEEE, 2012.

[13] R. Mesiar, A. Kolesárová, T. Calvo, and M. Komorníková, A review of aggregation functions, editor. Fuzzy Sets and Their Extensions: Representation, Aggregation and Models, H. Bustince et al. (Eds.), Springer, Berlin, 121-144, 2008.

[14] M. Mizumoto and K. Tanaka, Fuzzy sets of type2 under algebraic product and algebraic sum, Fuzzy Sets and Systems, 5:277-290, Elsevier, 1981.

[15] M. Mizumoto and K. Tanaka, Some properties of fuzzy sets of type-2, Information and Control, 31:312-340, Elsevier, 1976.

[16] Z. Takáč, Type-2 aggregation operators, editor, proceeding of the $8^{\text {th }}$ conference of the European 
Society for Fuzzy Logic and Technology (EUSFLAT 2013), pages 165-170, September 11-13, Milano (Italy), 2013.

[17] Z. Takáč, Aggregation of fuzzy truth values, Information Science, 271:1-13, Elsevier, 2014.

[18] C. Walker and E. Walker, Some general comments on fuzzy sets of type-2, International Journal of Intelligence Systems, 24:62-75, Willey, 2009.

[19] C. Walker and E. Walker, The algebra of fuzzy truth values, Fuzzy Sets and Systems, 149:309-347, Elsevier, 2005.

[20] C. Walker and E. Walker, T-norms for type-2 fuzzy sets, editor, proceeding IEEE Int. Conf. Fuzzy Syst., pages 1235-1239, Vancouver (Canada), 2006.

[21] R. Yager, Aggregation operators and fuzzy systems modeling, Fuzzy Sets and Systems, 67, no. 2:129145, Elsevier, 1994.

[22] R. Yager, Quantifier guided aggregation using OWA operators, International Journal of Intelligence Systems, 11, no. 1:49-73, Willey, 1996.

[23] L. Zadeh, Fuzzy sets, Information and Control, 20:301-312, Elsevier, 1965.

[24] L. Zadeh, The concept of a linguistic variable and its application to approximate reasoning, Information Science, 8:199-249, Elsevier, 1975.

[25] S. Zhou, F. Chiclana, R. John, and J. Garibaldi, Type-1 OWA operators for aggregating uncertain information with uncertain weights induced by type-2 linguistic quantifiers, Fuzzy Sets and Systems, 159, no. 24:3281-3296, Elsevier, 2008. 